cerebral hypomyelination, and cerebellar atrophy had nucleotide alterations in the folate receptor 1 gene, FOLRl. These included novel pathogenic alleles in 4, one splice mutation and 3 missense mutations, with absence of cellular binding of folic acid. The molecular studies did not consistently explain the phenotypic variations among patients with cerebral folate transport deficiency, and additional factors must be contributory. Most patients had frequent myoclonic seizures or infantile spasms and benefited from oral folinic acid. (Folic acid must be converted to biologically active 5-MTHF and folinic acid is preferred). Response occurred within 2 months with reduced frequency of seizures and improved motor skills. The EEG showed a slow background rhythm and multifocal epileptiform activity. MRI showed delayed or hypomyelination of cerebral white matter and cerebellar atrophy. (Grapp M, Just IA, Linnankivi T, et al. Molecular characterization of folate receptor 1 mutations delineates cerebral folate transport deficiency. Brain 2012 Jul;135(Pt 7):2022-31). (Respond: Dr Robert Steinfeld. E-mail: rsteinfeld@med.unigoettingen.de).

COMMENT. Cerebral folate deficiency is a progressive neurologic disorder of childhood amenable to treatment with folinic acid. Mutations in the FOLR1 gene are the most common and severe etiology of cerebral folate deficiency, and are associated with a characteristic phenotype with early onset ( $<3$ years), developmental delay, ataxia, and seizures often resembling infantile spasms. Seizures may prove refractory to oral folinic acid, and parenteral or rarely intrathecal administration may be required. (McFarland $\mathrm{R}$. Scientific commentaries. Cerebral folate deficiency - mishaps and misdirection. Brain 2012 Jul;135(Pt 7):2002-3).

\title{
EEG AND SEIZURE MANIFESTATIONS OF CEREBRAL FOLATE DEFICIENCY
}

Researchers from Duke University Medical Center, Durham, NC report the EEG and seizure manifestations in 2 patients with folate receptor autoimmune antibodymediated primary cerebral folate deficiency. Case 1, a boy presented at age 6 months with flexion spasms of the trunk and extension of the arms occurring multiple times each day. The seizures were diagnosed as infantile spasms and the EEG showed hypsarrhythmia. Treatment with various anticonvulsant drugs and ACTH $80 \mathrm{U} / \mathrm{m} 2$ provided only minor seizure control. Decreased CSF 5-MTHF and elevated serum folate receptor antibodies discovered at age 3.25 years were consistent with an autoimmune etiology of cerebral folate deficiency. Treatment with folinic acid started at $1 \mathrm{mg} / \mathrm{kg} / \mathrm{day}$ resulted in marked clinical and EEG improvement. MRI revealed diffuse brain volume loss. Case 2, a girl aged 4 years had a history of developmental delay and tonic seizures that increased in frequency after age 5 years. She had progressive regression with language deficits, hand wringing, and gait difficulty. Tests for Rett syndrome and related disorders were negative. With increasing age her seizures were multiple and refractory. The EEG showed subclinical electrical status epilepticus during sleep, covering $>85 \%$ of the slow wave sleep background. Cerebral folate deficiency was identified at age 16 years, and seizure frequency and intensity improved with folinic acid initiated at a dosage of $0.5 \mathrm{mg} / \mathrm{kg} /$ day. The EEG showed less frequent multifocal spikes, and electrical status epilepticus during sleep resolved. (Steele SU, Cheah SM, Veerapandiyan A, Gallentine 
W, Smith EC, Mikati MA. Electroencephalographic and seizure manifestations in two patients with folate receptor autoimmune-mediated primary cerebral folate deficiency. Epilepsy Behav 2012 Aug;24(4):507-12) (Response: MA Mikati MD. E-mail: mohamad.mikati@duke.edu).

COMMENT. Patients with developmental regression, refractory seizures or spasms, and EEG showing hypsarrhythmia or electrical status epilepticus during sleep should be tested for cerebral folate deficiency and considered for treatment with folinic acid. The authors list seizure onset during the first 2 years, tonic, myoclonic-astatic, absence, or generalized tonic-clonic seizures, and an EEG showing generalized spikeslow waves and multifocal spikes as important in the index of suspicion of this disorder.

\section{VASCULAR DISORDERS}

\section{STROKE RECURRENCE IN CONGENITAL HEART DISEASE}

Researchers at the Hospital for Sick Children, Toronto, Canada identified 135 patients with congenital heart disease diagnosed with arterial ischemic stroke during 1992-2008 and registered in the Canadian Pediatric Stroke Registry-Toronto site. Of the total cohort with sentinel stroke, $19(14 \%)$ had a recurrence. Of $78(58 \%)$ with neonatal sentinel stroke, $7(9 \%)$ had a stroke recurrence. Ten years following a sentinel stroke, $27 \%$ had suffered a stroke recurrence, $26 \%$ had died, and $47 \%$ were alive without recurrence. Age at sentinel stroke was $0.5 \mathrm{yr}$ (range 0.1-17.0). Stroke recurrence risk was highest immediately following the sentinel stroke and decreased over time. At time of recurrence, $50 \%$ were receiving anticoagulation. Recurrence risk factors included a mechanical valve, prothrombotic condition, and an acute infection at time of sentinel stroke. Hazard of mortality after recurrence was similar to mortality after sentinel stroke. More aggressive secondary prophylaxis in the early poststroke period may be indicated in patients at increased risk. (Rodan L, McCrindle BW, Manlhiot C, et al. Stroke recurrence in children with congenital heart disease. Ann Neurol 2012 Jul;72(1):103-11). (Respond: Gabrielle deVeber MD, Hospital for Sick Children, 555 University Ave, Toronto, Canada M5G 1XB. E-mail: gabrielle.deveber@sickkids.ca).

COMMENT The Toronto team has demonstrated the relative safety of anticoagulant therapy in 123 children with arterial ischemic stroke, with a $4 \%$ risk of intracranial hemorrhage. (Schechter T et al. Blood 2012 Jan 26;119(4):949-56).

\section{NEONATAL DISORDERS}

\section{SURFACTANT AND NEONATAL ELECTROENCEPHALOGRAM}

Researchers at the University of California San Diego evaluated the effects of endotracheal intubation and surfactant on the neonatal brain using a 3-channel neonatal EEG. Surfactant administration was associated with brain wave suppression on EEG in $18(62 \%)$ of 29 infants treated. ( $<0.008)$. Nine infants exhibited EEG suppression during 